\title{
Theoretical investigations on the energy relaxation in two- dimensional GaN systems
}

\author{
M. Tas* and B. Tanatar \\ Department of Physics, Bilkent University, Ankara 06800, Turkey \\ Received 30 July 2006, revised 18 August 2006, accepted 18 August 2006 \\ Published online 7 February 2007
}

PACS 63.20.Kr, 72.20.Ht, 73.50.Fq, 73.63.Hs

We theoretically study the energy relaxation of hot electrons via LO-phonon emission in two-dimensional GaN systems. We employ a model in which electrons and the lattice are in equilibrium separately, and the effective electron temperature is much larger than the lattice temperature. We include the dynamical screening of electrons, electron-phonon interactions reduced phonon self-energy correction, the hot-phonon, and the finite-size effects. The power loss to acoustic phonons is also considered.

In the last few years, a great deal of experimental and theoretical attention has focused on the direct, large band gap III-V nitride semiconductors GaN, AlN and InN. The large band gap leads to high breakdown voltages and electric fields, good thermal conductivity, low noise generation, high power and high temperature operation. All of these properties make these structures desirable for electronic and optoelectronic device applications [1-7]. These devices work mostly under high electric field, which brings about substantial heating and decrease in their performance. For desired efficiency in device applications and good explanation of the experimental results, the electronic processes behind the transport properties of nitrate heterostructures should be understood clearly.

The electrons in a bulk or quantum well become energetic (or hot) when a high electric field is applied, and the thermal equilibrium between the electron gas and the lattice is destroyed. The electrons relax by losing their excess energy to the lattice. This process follows several steps. First, the electrons having enough energy emit longitudinal optical (LO) phonons, Then, the emitted LO phonons decay into acoustic phonons and the excess energy drains out to the lattice. Finally, the electron gas and the lattice reach equilibrium separately at different temperatures, $T$ and $T_{L}$, respectively, coupled only weakly through electron-phonon interaction. In the steady state the input power is equal to the power loss to the lattice. At high electron temperatures the dominant mechanism for energy relaxation is emitting LO phonons. However, there are several parameters affecting the power loss of hot electrons: the electron-phonon coupling, electronic screening, hot-phonon and finite-thickness effects. Throughout the years, there has been a large amount of theoretical effort to explain the experimental results for the power loss of electrons in semiconductor bulk and lower dimensional structures, especially GaAs based quantum wells [8-17].

In order to understand the power loss mechanism of hot electrons in GaN quantum wells, we made a detailed theoretical investigation. We included the dynamical screening of electrons within the finitetemperature random-phase approximation (RPA). Phonon self-energy renormalization due to the electronphonon coupling, hot-phonon effect, impurity and the finite-thickness effect are also considered. In polar semiconductors like GaN the piezoelectric coupling to acoustic phonons is quite important at low temperatures. Thus, we included the deformation potential and piezoelectric coupling of acoustic phonons to electrons. On the other hand, for ease of numerical computations we made several acceptable physical approximations. The two-dimensional (2D) electrons are assumed to interact with bulk phonons. We employed the effective electron-temperature model, which assumes $T>T_{L}$. Taking $T_{L}=0$, the power loss

* Corresponding author: e-mail: tasm@fen.bilkent.edu.tr, Phone: +90 312290 2514, Fax: +90312 2664579 


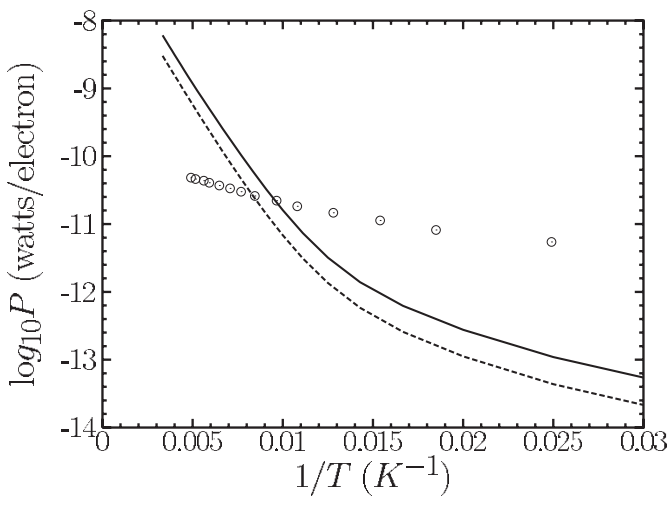

Fig. 1 The power loss per electron as function of electron temperature $T$ with renormalized phonon propagator. The solid (dashed) line shows the results without (with) the finite-size effects. The power loss to acoustic phonons is ignored. The circles are the experimental data of Fig. 4 of Ref. [5].

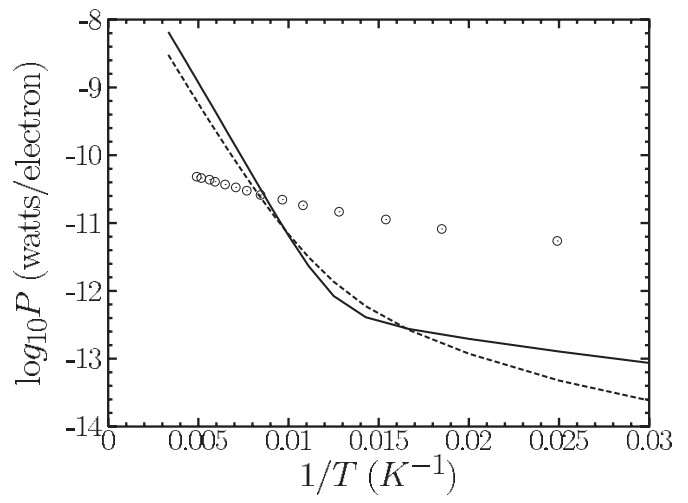

Fig. 2 The power loss per electron as function of electron temperature $T$. The solid (dashed) line shows the results with bare (renormalized) phonon propagator. The power loss to acoustic phonons, and the finite-size effects are included. The circles are the experimental data of Fig. 4 of Ref. [5].

per electron is [17]

$$
P=\frac{1}{N} \sum_{q} \int \frac{d \omega}{\pi} \hbar \omega n_{T}(\omega) M_{q}^{2} \operatorname{Im} \chi(q, \omega) \operatorname{Im} D(q, \omega),
$$

where $n_{T}(\omega)$ is the Bose occupation number at the electron temperature, $M_{q}$ is the Fröhlich electronphonon coupling strength, $\chi$ is the finite-temperature reducible RPA polarizability of the electron gas, and $D$ is the interacting phonon propagator. Here, the phonon self-energy correction due to the electron-phonon interaction, and the dynamical screening are included through $D$ and $\chi$, respectively. The experiments we are interested in have been done with GaN/AlGaN high electron mobility transistor (HEMT) structures of typical width of 25-30 nm. The finite width of the quantum well weakens the strength of the electronelectron interaction and the coupling of the electrons to LO phonons. These effects are included by replacing the $2 \mathrm{D}$ Coulomb interaction between electrons $V_{q}$ by $f_{q} V_{q}$ in $M_{q}^{2}$ and $\chi$, where $f_{q}$ is an appropriate form factor [12]. Ignoring the mode coupling effects replaces $D$ by the bare phonon propagator. Then, the power loss relation becomes

$$
P=\frac{1}{N} \sum_{q} \hbar \omega_{q} R_{q} n_{T}\left(\omega_{q}\right), \quad R_{q}=-2 \hbar^{-1} M_{q}^{2} \operatorname{Im} \chi\left(q, \omega_{q}\right),
$$

where $R_{q}$ is the scattering rate.

The emitted LO phonons do not decay into acoustic phonons immediately. Because they have a finite lifetime, they can be reabsorbed by the electrons before they decay. This phenomena is known as hotphonon effect, which decreases the power loss. The expression for the power loss including the hot-phonon effect has been found as [14]

$$
P=\hbar \omega_{L O} n_{T}\left(\omega_{L O}\right) \frac{1}{N} \sum_{q} \frac{R\left(q, \omega_{L O}\right)}{1+\tau_{p h} R\left(q, \omega_{L O}\right)},
$$

where $\tau_{p h}$ is the phonon lifetime, and $R\left(q, \omega_{L O}\right)$ is the scattering rate as defined above. This equation reduces to (2) for vanishing phonon lifetime when $\omega_{q}=\omega_{L O}$.

In our numerical calculations, we used the material constants of GaN. Our results are compared with the experimental data of Mazzucato et al. [5] in Figs. 1-3. Details of the theoretical models are given in 

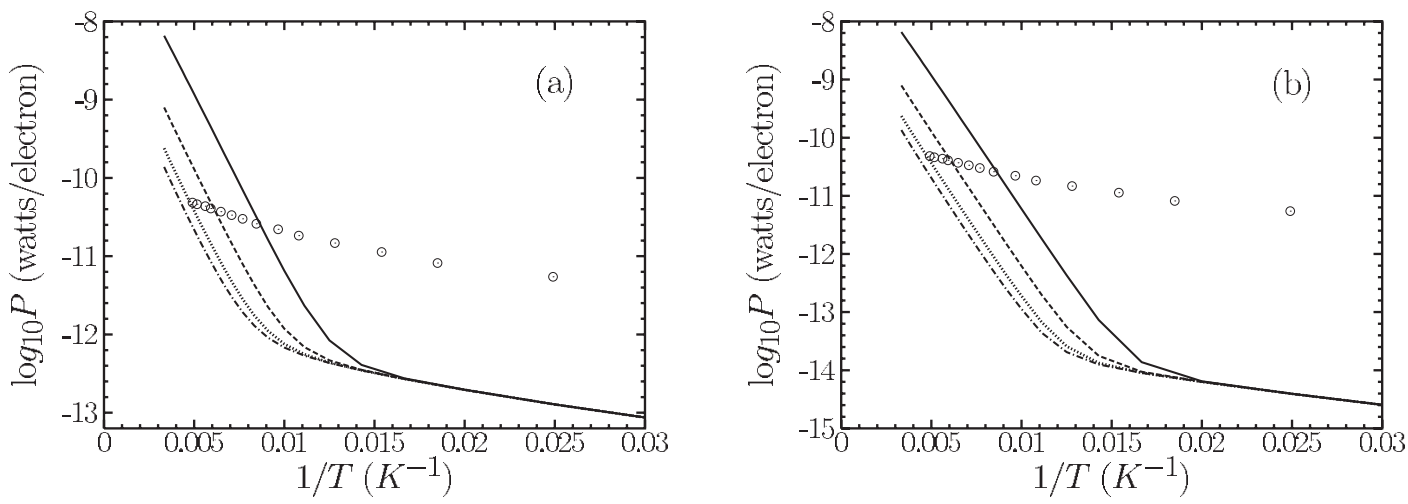

Fig. 3 The power loss per electron as function of electron temperature $T$ for different phonon lifetimes $\tau_{p h}$ : 0 ps (solid line), 1 ps (dashed line), 5 ps (dotted line), and 10 ps (dashed-dotted line). In (a) the mode coupling is ignored. However, in (b) it is considered. In both figures the power loss to acoustic phonons and the finite-size effects are included. The circles are the experimental data of Fig. 4 of Ref. [5].

the figure captions. Overall, the many-body approaches that assume the electron temperature model used in this work do not satisfactorily describe the data. The sophisticated theoretical modelling presented here hass been found successful in previous studies [18]. One possible source of discrepancy is the accuracy with which the electron density is determined from mobility in the experiments [5]. It has also been noted that with increasing electric field, the measured power loss would deviate from theoretical values because of enhanced carrier capture. These suggest that there is need for further theoretical investigation for better agreement with experiments.

Acknowledgements We thank Dr. S. Mazzucati for sending us the data of Fig. 4 in Ref. [5]. M. T. would like to thank TUBITAK for financial support. B. T. is supported by TUBITAK, TUBA, and British Council.

\section{References}

[1] V. Sivakumar and U. V. Varadaraju, J. Electrochem. Soc. 153, H54 (2006).

[2] D. Zanato et al., phys. stat. sol. (c) 2, 3077 (2005).

[3] D. Zanato et al., Semicond. Sci. Technol. 19, 1024 (2004).

[4] D. Zanato, N. Balkan, G. Hill, and W. J. Schaff, Superlattices Microstruct. 36, 455 (2004).

[5] S. Mazzucato et al., Physica B 314, 55 (2002).

[6] N. Balkan et al., J. Phys.: Condens. Matter 14, 3457 (2002).

[7] A. Bonfiglio et al., J. Appl. Phys. 87, 2289 (2000).

[8] Sh. M. Kogan, Fiz. Tverd. Tela 4, 2474 (1963) [Sov. Phys.-Solid State 4, 1813 (1963)].

[9] E. Conwell, High Field Transport in Solids (Academic Press, New York, 1963).

[10] B. K. Ridley, J. Phys. C: Solid State Phys. 15, 5899 (1982).

[11] S. Das Sarma, J. K. Jain, and R. Jalabert, Phys. Rev. B 37, 1228 (1988).

[12] S. Das Sarma, J. K. Jain, and R. Jalabert, Phys. Rev. B 37, 4560 (1988).

[13] S. Das Sarma, J. K. Jain, and R. Jalabert, Phys. Rev. B 37, 6290 (1988).

[14] S. Das Sarma, J. K. Jain, and R. Jalabert, Phys. Rev. B 41, 3561 (1989).

[15] P. Tripathi and B. K. Ridley, Phys. Rev. B 66, 195301 (2002).

[16] C. Bulutay, B. K. Ridley, and N. A. Zakhleniuk, Phys. Rev. B 68, 115205 (2003).

[17] L. Zheng and S. Das Sarma, Phys. Rev. B 54, 2751 (1996).

[18] N. M. Stanton et al., J. Appl. Phys. 89, 973 (2001). 